\title{
Binding to sulfatide and enterotoxicity of various Escherichia coli STb mutants
}

\author{
Vincent Labrie, Hans-Erick Beausoleil, Josée Harel and J. Daniel Dubreuil
}

Groupe de Recherche sur les Maladies Infectieuses du Porc, Département de Pathologie et

Microbiologie, Faculté de Médecine Vétérinaire, Université de Montréal, 3200 Sicotte, CP 5000, Saint-Hyacinthe, Québec, Canada J2S 7C6
Author for correspondence: J. Daniel Dubreuil. Tel: +1 4507738521 ext. 8433. Fax : +1 4507788108. e-mail: daniel.dubreuil@umontreal.ca

\begin{abstract}
Binding of the $\mathbf{4 8}$ amino acid polypeptide of the mature heat-stable Escherichia coli enterotoxin b (STb) to the functional receptor sulfatide (SFT) constitutes the first step in inducing secretory diarrhoea in the intestinal lumen of animals. The NMR structure of this toxin dictated the choice of amino acids for site-directed mutagenesis to delineate the binding site of STb to SFT. Amino acids facing the solvent either in the loop or the hydrophobic $\alpha$-helix were selected. Seventeen site-specific mutants of STb toxin were produced and purified by high-pressure liquid chromatography. Enterotoxicity of the 17 mutants was determined using a rat loop assay and binding was evaluated using a microtitre plate binding assay. Both hydrophobic and electrostatic interactions are important for STb attachment. When mutations (F37K, $141 \mathrm{~S}$ and M42S) were introduced into the hydrophobic $\alpha$-helix to lessen hydrophobicity, binding activity and enterotoxicity decreased by more than sixfold. The loop defined by C21 and C36 also made specific contributions. Mutants generated at basic residues (K22, K23 and R29) within this region exhibited both reduced binding activities and reduced toxic activities. For all STb mutants constructed and analysed, when binding to SFT was reduced, a reduction in toxicity equivalent or greater was noted, indicating that binding to SFT is a step that precedes the toxic effect observed for STb toxin. Significantly, when the negatively charged D30 was substituted for either alanine or valine, the binding to SFT was about twice that of native STb, whereas the enterotoxicity was reduced by half.
\end{abstract}

Keywords: enterotoxin b, mutagenesis, binding, ETEC

\section{INTRODUCTION}

Enterotoxigenic Escherichia coli (ETEC) strains cause severe diarrhoeal diseases in humans and animals. Two classes of enterotoxins are responsible for the pathogenesis: heat-labile enterotoxins (LTI and LTII), which are structurally and functionally related to the cholera toxin (Kaper et al., 1995; Spangler, 1992), and the heatstable toxins (STs). Two subtypes, sharing no homology at the nucleotide or amino acid levels, are identified as heat-stable toxins, namely STa and STb (Dubreuil, 1997). These low-molecular-mass toxins are extracellular enterotoxins; STb is methanol-insoluble whereas STa is not. STa is an 18 or 19 aa acidic peptide that binds to particulate transmembrane guanylate cyclase C (GC-C). This binding leads to an increase in cGMP and eventually the opening of the cystic fibrosis

Abbreviations: $C D$, circular dichroism; SFT, sulfatide. transmembrane regulator (CFTR) channel responsible for chloride secretion (Goldstein et al., 1994).

In contrast to LT and STa toxins, there is no evidence that $\mathrm{STb}$ is associated with an intracellular elevation of cyclic nucleotide (Kennedy et al., 1984; Peterson \& Whipp, 1995; Weikel \& Guerrant, 1985). Instead, in vitro studies have shown that the intestinal secretion pathway involves the activation of a GTP-binding regulatory protein $\left(\mathrm{G}_{\alpha \mathrm{i} 3}\right.$ protein) sensitive to pertussis toxin. This action results in an intracellular calcium elevation through a receptor-dependent ligand-gated $\mathrm{Ca}^{2+}$ channel (Dreyfus et al., 1993). The ensuing high level of intracellular $\mathrm{Ca}^{2+}$ is presumably involved in activation of a calmodulin-dependent protein kinase II (CaMK II) which could open an undetermined ionic channel (Fujii et al., 1997). In addition, intracellular concentration of $\mathrm{Ca}^{2+}$ may regulate phospholipases $\mathrm{A}_{2}$ and $\mathrm{C}$, which catalyse the release of arachidonic acid from the membrane phospholipids, and ultimately the 


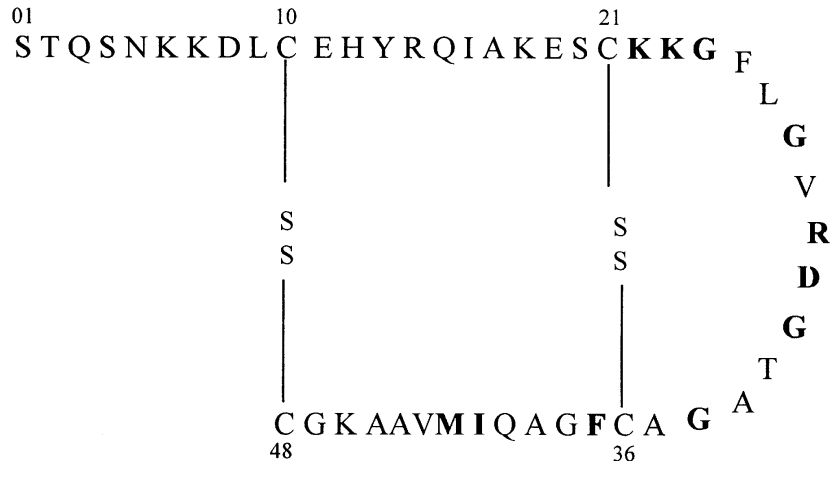

Fig. 1. Amino acid sequence of mature STb enterotoxin. C10 to K23 represents the stretch forming the amphipathic $\alpha$-helix and G38 to A44 represents the hydrophobic $\alpha$-helix. Bold letters represent amino acids targeted for mutagenesis.

Table 1. Biological activity of STb mutants as determined in previous studies

\begin{tabular}{|lcl|}
\hline Mutations & $\begin{array}{c}\text { Biological activity } \\
(\%)\end{array}$ & \multicolumn{1}{c|}{ Reference } \\
\hline STb & $100 \cdot 0$ & \\
K22L & $10 \cdot 0$ & Fujii et al. $(1994)$ \\
K23T & $10 \cdot 0$ & Fujii et al. (1994) \\
K22I, K23N & $2 \cdot 5$ & Fujii et al. $(1994)$ \\
R29I & $50 \cdot 0$ & Fujii et al. $(1994)$ \\
R29S & $12 \cdot 7$ & Dreyfus et al. $(1992)$ \\
D30V & $39 \cdot 0$ & Dreyfus et al. $(1992)$ \\
\hline
\end{tabular}

formation of prostaglandin $\mathrm{E}_{2}\left(\mathrm{PGE}_{2}\right)$ known as a secretory agent (Fujii et al., 1995; Hitotsubashi et al., 1992; Peterson \& Whipp, 1995). Recently, our laboratory demonstrated in vivo the functionality of sulfatide (SFT) as a receptor for STb (Rousset et al., 1998). We determined its affinity constant $\left(K_{\mathrm{d}}\right)$ between 2 and $6 \mu \mathrm{M}$ (Beausoleil \& Dubreuil, 2001). Unsolved remain the steps between the attachment of $S T b$ to its receptor and the activation of the $G$ protein presumably involved in signal transduction.

$\mathrm{STb}$ toxin synthesized as a 71 aa precursor (Fig. 1) undergoes cleavage of the $\mathrm{N}$-terminus signal sequence (residues 1-23) (Kupersztoch et al., 1990). This process results in a 48 aa polypeptide of $5200 \mathrm{Da}, \mathrm{pI} 9 \cdot 6$ (Handl et al., 1993). Maturation also involves the formation of two disulfide bridges: between $\mathrm{C} 10$ and $\mathrm{C} 48$ and between $\mathrm{C} 21$ and C36. Both disulfide bridges are important for stabilization of the molecule's tertiary structure (Sukumar et al., 1995) and are indispensable for enterotoxicity (Arriaga et al., 1995; Okamoto et al., 1995). NMR studies of STb have established a structure having two antiparallel $\alpha$-helix motifs separated by a loop. An amphipathic helix between residues 10 and 22 exposes several polar side chains to the solvent. Facing this is a hydrophobic helix extending from residue 38 to 44. The glycine-rich loop presents some amino acids that previous mutagenesis studies have demonstrated as being important in enterotoxicity (Sukumar et al., 1995).

Mutagenesis studies (Table 1) (Dreyfus et al., 1992; Fujii et al., 1994) have reported the relative importance of some amino acids in enterotoxicity, but none have been conducted in order to evaluate their implication in the attachment to the SFT receptor. Consequently, nothing is known about the possible segregation between the binding domain and the domain responsible for enterotoxicity. Since it has been shown that the glycine-rich motif could participate in various functions in proteins, such as in the flexible arm of the monomeric $\alpha$ haemolysin of Staphylococcus aureus allowing its membrane insertion (Ward et al., 1994), it was of interest to further investigate the glycine-rich loop of STb between C21 and C36. Likewise, the hydrophobic region of the $\mathrm{STb} \alpha$-helix shares some structural properties with the $S$. aureus pore-forming $\alpha$-toxin, which also possesses amphipathic and hydrophobic $\alpha$-helices involved in pore formation (Lala \& Raja, 1995; Ward et al., 1994).

Although a small polypeptide, STb has primary and secondary structures possessing some features that could play important roles in binding to a receptor and either cell penetration or signal transduction involving a $G$ protein. Our objective was to confirm or refute the role of selected amino acids in enterotoxicity and also to delineate the amino acids involved in the interaction of $\mathrm{STb}$ with SFT.

\section{METHODS}

Oligonucleotide-directed mutagenesis and DNA sequencing. Oligonucleotide-directed site-specific mutagenesis was performed using the USE Mutagenesis Kit purchased from Pharmacia Biotech. The mutagenic primers (Table 2) used to generate point mutations were synthesized by Gibco-BRL. The plasmid pMal-STb, already described (Bossé et al., 1993), served as template for simple and double mutations. The mutated plasmid pMal-STb-R29A served for one double (K23A-R29A) mutation. All mutants were confirmed by automated sequencing with the RmalE primer 5'-GTAACGCCAGGGTTTTCC- $3^{\prime}$ at the DNA sequencing core facility (University of Maine, Orono, USA) by using an ABI model 373A stretch DNA sequencer.

Purification. To obtain pure STb mutants and native STb enterotoxin, the peptides were produced according to a previously described method (Handl et al., 1993). Briefly, E. coli strain HB101, harbouring the plasmid pMal-STb with or without a selected mutation, highly expressed the fusion protein, MBP-STb or MBP-mutated STb, under the control of $\mathrm{P}_{\text {tac }}$. Following the recovery from osmotic shock, the fusion protein was affinity purified by using an amylose resin (New England Biolabs) and cleaved with the protease factor Xa. The cleaved material was loaded on a $\mathrm{C}_{8}$ reverse-phase microbore column (Applied Biosystems) and eluted with an acetonitrile gradient (model 130A Separation System, Applied Biosystems). Purified peptides were lyophilized and stored at $-20^{\circ} \mathrm{C}$. The purity of the toxin and mutants was routinely verified by $\mathrm{N}$-terminal sequence analysis using Edman degradation (Applied Biosystems model 470A gas-phase sequencer) as previously described (Handl et al., 1993).

Circular dichroism analysis. Circular dichroism (CD) measurements were carried out on a Jasco J-710 spectropolarimeter. 
Table 2. Synthetic mutagenic primers used in this study

\begin{tabular}{|c|c|}
\hline Mutations & Oligonucleotide sequence* \\
\hline $\mathrm{K} 22 \rightarrow \mathrm{A}$ & СССТАAАAАACСТTTCGCACAACTTTCCTTGGC \\
\hline $\mathrm{K} 23 \rightarrow \mathrm{A}$ & 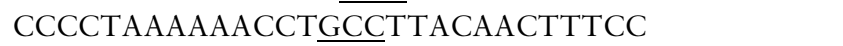 \\
\hline $\mathrm{K} 22 \rightarrow \mathrm{A}, \mathrm{K} 23 \rightarrow \mathrm{A}$ & ССССТАAАAАAССТGCCGCACAACTTTCCTTGGC \\
\hline $\mathrm{G} 24 \rightarrow \mathrm{A}$ & ССССТАAAAACGCTTTCETTACAACTTTCC \\
\hline $\mathrm{G} 27 \rightarrow \mathrm{P}$ & CCATCTCTAACCGGTAAAAAACC \\
\hline $\mathrm{R} 29 \rightarrow \mathrm{A}$ & GCAGTACCATCTGCAACCCСТAAAAAACC \\
\hline \multirow[t]{2}{*}{$\mathrm{K} 23 \rightarrow \mathrm{A}, \mathrm{R} 29 \rightarrow \mathrm{A}$} & CСССТАAAAAACСТGCCTTACAACTTTCC \\
\hline & GCAGTACCATCTGCAACCCCTAAAAAACC \\
\hline $\mathrm{D} 30 \rightarrow \mathrm{A}$ & CCAGCAGTACCA $\overline{G C T C T A A C C C C ~}$ \\
\hline $\mathrm{D} 30 \rightarrow \mathrm{V}$ & CCAGCAGTACCA $\underline{\bar{A}} \mathrm{CTCTAACCCC}$ \\
\hline $\mathrm{G} 31 \rightarrow \mathrm{A}$ & GCTCCAGCAGTA $\underline{\bar{G}}$ CATCTCTAACCC \\
\hline $\mathrm{G} 34 \rightarrow \mathrm{A}$ & CCAAAGCATGCTGCAGCAGTACC \\
\hline $\mathrm{F} 37 \rightarrow \mathrm{K}$ & CCATTATTTGGGCGCCTTTACATGCTCCAGC \\
\hline $\mathrm{F} 37 \rightarrow \mathrm{D}$ & CCATTATTTGGGCGCCATCACATGCTCCAGC \\
\hline $\mathrm{I}-41 \rightarrow \mathrm{S}$ & GCTGCAACCATCGATTGGGCGCCAAAGC \\
\hline $\mathrm{M} 42 \rightarrow \mathrm{S}$ & CCTTTTGCTGCAACGCTTATTTGGGCGCC \\
\hline $\mathrm{M} 42 \rightarrow \mathrm{R}$ & CCTTTTGCTGCAACGCGTATTTGGGCGCC \\
\hline $\mathrm{I} 41 \rightarrow \mathrm{E}, \mathrm{M} 42 \rightarrow \mathrm{R}$ & CCTTTTGCTGCTGCAACGCGTTCTTGGGCGCCAAAGC \\
\hline
\end{tabular}

* The changed bases on the antisense strand of the plasmid Pmal-STb are underlined.

Far-UV (300-185 nm) runs were conducted in a $0 \cdot 1 \mathrm{~cm}$ cell maintained at room temperature. Concentrations of STb and mutant STb were adjusted to $0.75 \mathrm{mg} \mathrm{ml}^{-1}$ in phosphate buffer $0.02 \mathrm{M}(\mathrm{pH} 7 \cdot 2)$. The data collected were computer-averaged for ten scans, baseline subtracted, and smoothed.

Microtitre plate binding assay. U-bottom polystyrene microtitre plates (Serocluster, Costar) were coated by overnight evaporation of $50 \mu \mathrm{l}$ methanol-diluted SFT (500 ng) (Sigma). Free sites were blocked with $200 \mu \mathrm{l}$ per well phosphate-buffer (PBS)-casein $(0.3 \%, \mathrm{w} / \mathrm{v}) \mathrm{pH} 7 \cdot 4$ for $2 \mathrm{~h}$ at room temperature. Purified STb toxin (500 ng) or mutant STb in $50 \mu \mathrm{l}$ PBS $(0 \cdot 1 \mathrm{M}$ phosphate containing $150 \mathrm{mM} \mathrm{NaCl}) \mathrm{pH} 7 \cdot 0$ were added per well in triplicate for $1 \mathrm{~h}$ at $37^{\circ} \mathrm{C}$. After three washes with PBScasein, each well was filled with $50 \mu \mathrm{l}$ rabbit monospecific anti$\mathrm{STb}$ antibodies diluted 1:1000 in PBS-casein for $1 \mathrm{~h}$ at $37^{\circ} \mathrm{C}$. The plates were washed three times with PBS-casein. Goat anti-rabbit IgG conjugated to horseradish peroxidase (Jackson Immunoresearch) was diluted 1:3000 in PBS-casein and $50 \mu \mathrm{l}$ was applied to each well before incubation at $37^{\circ} \mathrm{C}$ for $1 \mathrm{~h}$. Following three washes with PBS-casein, $50 \mu \mathrm{l}$ chromogenic substrate ABTS [2,2'-azino-bis(3-ethylbenzthiazoline-6-sulfonic acid)] (Sigma) $(0.4 \mathrm{mM})$ dissolved in citrate buffer $\mathrm{pH} 4 \cdot 0$ containing $0.5 \mathrm{mM} \mathrm{H}_{2} \mathrm{O}_{2}$ was added. The absorbance was measured at $410 \mathrm{~nm}$ using an automatic ELISA reader (model MR5000, Dynatech Laboratories).

Triplicates of methanol with and without SFT served as negative controls to evaluate the background after the blocking step and the reaction of anti-STb antiserum with SFT, respectively. For analysis, the mean absorbance obtained for these wells was subtracted from the mean absorbance for $\mathrm{STb}$ or each mutant tested. The value for $\mathrm{STb}$ was considered as $100 \%$ binding and all the data were calculated relative to this value. The final value represents the mean of at least two assays.

Ligated rat jejunal loop assay. Enterotoxicity studies were conducted using a ligated rat jejunal loop assay as described previously (Dubreuil et al., 1991). Briefly, 6- to 8-week-old white male rats (Sprague Dawley CD; Charles River Ltd) were fasted for $48 \mathrm{~h}$ and anaesthetized. The small intestine was exteriorized following a midline abdominal incision and rinsed for $5 \mathrm{~min}$ with $8 \mathrm{ml} 0.85 \%$ saline solution containing $300 \mu \mathrm{g}$ soybean trypsin inhibitor (Boehringer Mannheim) $\mathrm{ml}^{-1}$. A series of seven 5 -cm-long ligated segments (loops) was made in the jejunum. The posterior $30 \%$ of the small intestine starting at the caecum was not used. Each loop received $5 \mu \mathrm{g}$ test material in $500 \mu \mathrm{l} 20 \mathrm{mM}$ Tris/ $\mathrm{HCl}$ buffer ( $\mathrm{pH}$ 6.8) containing $300 \mu \mathrm{g}$ trypsin inhibitor $\mathrm{ml}^{-1}$. Loops receiving only the buffer were considered as the negative controls. The abdominal incision was closed. Three hours later, the rats were killed and the amount of fluid in each loop was measured. Results are expressed as the ratio of millilitres fluid accumulated per centimetre ligated jejunal segment. Each mutant was tested twice in each rat, varying the position among the loops, and at least six rats were used for each mutant. To minimize the effect of sample position, two mutants and two STb-positive controls were alternated in each rat. Samples were always separated by buffer (negative control).

For analysis, the mean of three negative controls was subtracted from the values of two positive controls (STb alone) and from two samples of each mutant tested, in the same rat. Finally, from these results the mean and standard deviation were calculated, omitting the highest and the lowest values. The value for STb was considered as $100 \%$ biological activity and all the data were transformed relative to this value.

\section{RESULTS}

\section{Rationale for production of mutant STb molecules}

To determine which STb mutants would contribute most to the understanding of the steps involved in the mechanism of action of this toxin, we first indexed all mutants produced in previous studies (Table 1). Those mutants were tested for biological activity, as indicated in Table 1, but were not assayed for binding to SFT. The 


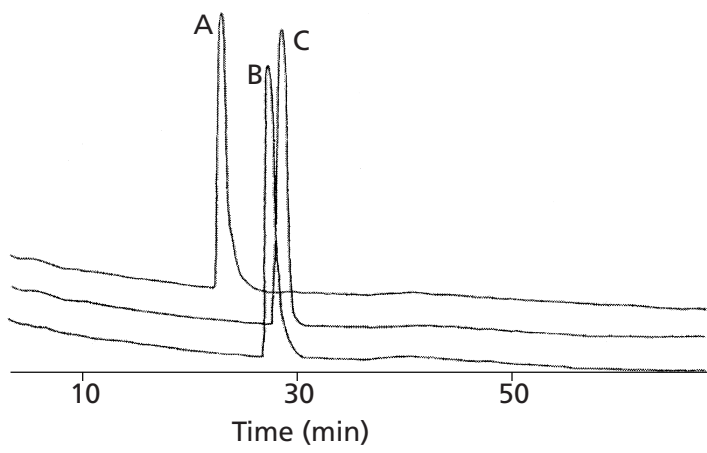

Fig. 2. HPLC chromatograms at $220 \mathrm{~nm}$ of purified STb and selected mutants. A, mutant $141 \mathrm{E}-\mathrm{M} 42 \mathrm{R}$, representing the group losing hydrophobicity upon mutation; B, native STb; C, mutant K22A-K23A, representing the group acquiring hydrophobicity upon mutation. Polypeptides were eluted with a gradient of acetonitrile containing $0.1 \%$ trifluoroacetic acid using a reverse-phase $\mathrm{C}_{8}$ microbore column.

sequence of STb (Fig. 1) and the available NMR model of STb (Sukumar et al., 1995) allowed us to more precisely target the amino acids facing the solvent at relevant positions and/or properties that could yield information on whether or not the binding and toxicity events are associated. On the basis of the information available and the aim of the study, 17 mutants were constructed and the mutagenic primers used in this study are shown in Table 2.

\section{Analysis of STb mutants by CD}

Properly oxidized disulfide bonds have been shown to be important for full STb enterotoxicity (Arriaga et al., 1995). In our study, we observed that STb attachment to SFT is also dependent on the presence of oxidized disulfide bonds. Upon $\beta$-mercaptoethanol treatment, the binding to SFT decreased to $10 \%$ (data not shown). Because important conformational changes induced by point mutations in $\mathrm{STb}$ could prevent the proper oxidation of cysteines in disulfide bridges, we analysed each mutant by measuring their CD spectra to confirm that all mutants retained their full structural integrity and in particular the presence of both disulfide bonds. Thus, the main chain structure of the STb enterotoxin, representing the $\alpha$-helix-loop- $\alpha$-helix pattern, appears not likely to be greatly affected by our site-directed mutagenesis. The $\mathrm{CD}$ spectra were similar to native $\mathrm{STb}$, with the exception of mutant M42S, which seems slightly different (data not shown).

\section{Hydrophobicity profiles of STb mutants}

As shown in Fig. 2, STb mutants were purified to homogeneity as demonstrated by the single elution peak using a $\mathrm{C}_{8}$ reverse-phase column. These chromatograms represent the hydrophobicity profiles of the toxin mutants generated. As expected, mutations changing a non-polar residue for a polar one in the hydrophobic $\alpha$ helix (F37, I41 and M42) diminished their retention time
$\left(t_{\mathrm{R}}\right)$ compared to native $\mathrm{STb}$ toxin. Almost all toxin mutants in the loop (C21-C36) were produced using the alanine-scanning procedure (Cunningham \& Wells, 1989). In this case, the $t_{\mathrm{R}}$ of the mutants was increased, indicating a predicted loss of polarity.

\section{Relative importance of the STb loop in attachment to SFT}

The microtitre plate binding assay, a technique derived from ELISA, allows a useful quantification of the affinity between a ligand and a receptor. In our study, we measured the interaction of different $\mathrm{STb}$ mutants with their known SFT receptor. This glycosphingolipid is a sulfated galactose residue joined to a ceramide moiety $\left[\mathrm{Gal}\left(3 \mathrm{SO}_{4}\right) \beta 1 \mathrm{Cer}\right]$. The evaluation of binding was done using the optimal parameters as determined for wildtype STb toxin. All mutants and STb toxin $(1 \mu \mathrm{g})$ reacted equally with the polyclonal monospecific anti$\mathrm{STb}$ serum (1/300) when blotted on Immunodyne membrane (Pall Biosupport Division). Quantitative binding analysis was determined by densitometric analysis as already described (Rousset et al., 1998) (data not shown). We first examined the charged amino acids present in the loop of STb. As shown in Fig. 3, a double mutation introduced in the contiguous lysines (K22K23) gave an average binding diminution of $91 \%$. When these lysines were mutated independently, relative attachment was respectively $52 \%$ and $54 \%$ of native STb. Arginine R29 was also changed for alanine, alone or comutated with K23. These modified toxins exhibited SFT-binding levels of $45 \%$ and $40 \%$, respectively. The double mutation K23A-R29A, however, did not show any decrease in SFT-binding affinity over that obtained with the single mutations K23A or R29A.

To establish the implication of the loop flexibility or simply the role of glycines in SFT binding, all glycines present in the loop (G24, -27 and -31) were subjected to site-directed mutagenesis. When the resulting mutant toxins were tested for their binding relative to the wildtype, values were reduced to 49,29 and $41 \%$, whereas for G34 the binding value was $118 \%$. Aspartate D30, also present in the loop, was changed for either alanine or valine, resulting in hyperattachment of $S T b$ polypeptide to SFT. The toxin with the D30A mutation bound less to SFT $(150 \%)$ than that with the D30V mutation $(210 \%)$.

\section{Importance of the hydrophobic $\alpha$-helix in attachment to SFT}

To reduce the hydrophobicity features of the C-terminal $\alpha$-helix, some highly non-polar residues pointing to the solvent (F37, I41 and M42) were targeted. None of them, even when mutated in combination, changed for negatively charged, positively charged or polar residues, showed a binding activity greater than $10 \%$ of native $\mathrm{STb}$, with the exception of mutant M42R, which expressed the same attachment to SFT as STb. Among all mutants, the double mutation I41E-M42R displayed the lowest affinity for the receptor $(3 \%)$. 


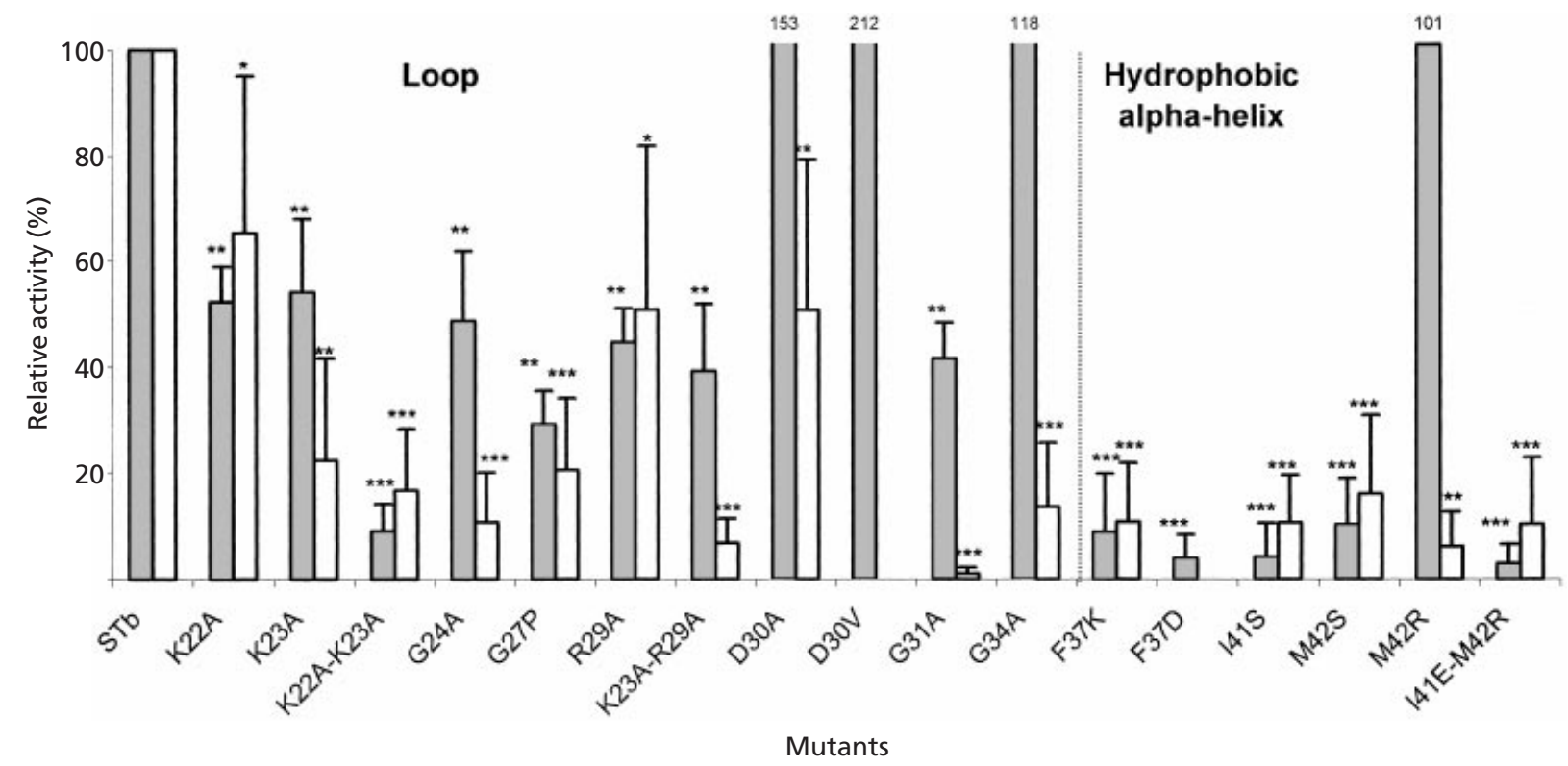

Fig. 3. Relative binding to SFT (grey columns) and enterotoxicity (white columns) of various STb mutants compared to STb native toxin. The left panel shows the results obtained for substitutions in the loop, the right panel, substitutions introduced in the C-terminal hydrophobic $\alpha$-helix. STb binding to SFT and enterotoxicity was taken as $100 \%$ and the relative activity of the mutants calculated as percentage. The binding activity of mutants over $100 \%$ are indicated as numbers at the top of the respective column. No experiments to determine the biological activity of mutants D30V and F37D were done. The standard deviations are shown. Mutants significantly different from STb were determined using Student's $t$-test: ${ }^{*} P<0.05 ; * * P<0.01 ; * * * P<0.001$.

\section{Relative biological activity}

The rat loop assay is a standard method that has proven to be useful for evaluating the toxicity of $\mathrm{STb}$ toxin. Among the 12 single mutants depicted in Fig. 3, the greatest decreases in toxicity were noted with three residues (F37K, I41S and M42R) located in the nonpolar helix and two located in the loop (G24A and G31A). The biological activity detected in rat loop assay for these five mutants averaged $11,11,6,11$ and $1 \%$, respectively. For the double mutation I41E-M42R, intestinal enterotoxicity $(11 \%)$ was similar to the single mutations. Inside the STb loop, diminution of the toxicity was observed with three single mutants assigned to basic residues of the loop (K22A, K23A and R29A) for which the enterotoxicity decreased to about 65,22 and $51 \%$, respectively. The consequences of combining substitutions were also studied. The double mutants K22A-K23A and K23A-R29A showed enterotoxicities of about 17 and $7 \%$, respectively. These values are lower than those for the corresponding single mutants. Small changes in toxicity were noted with D30A, for which the enterotoxicity was about $51 \%$. For mutants G27P and G34A, the enterotoxicity was reduced to 20 and $13 \%$, respectively.

\section{DISCUSSION}

In this study, we examined the role of specific amino acids of $\mathrm{STb}$ in recognition and binding to SFT and in the enterotoxicity determined in the rat loop assay. As the positively charged amino acids were previously shown to be important for toxicity, they were selected and tested in this study for binding to SFT. The loop region (C21-C36) and hydrophobic $\alpha$-helix were of special interest because they comprise respectively numerous charged and hydrophobic amino acids. As Sukumar et al. (1995) have determined the NMR solution structure for $\mathrm{STb}$, it was possible to make a critical choice about amino acids pointing into the solvent that could play a role in SFT binding and toxicity (Sukumar et al., 1995).

According to the highly basic pI (9.6) of STb and because of the anionic sulfate residue carried by SFT receptor, it is conceivable that some positively charged amino acid residues of $\mathrm{STb}$ could participate in bringing together and in binding to SFT. Previously, Fujii et al. (1994) demonstrated that the lysine residues K22 and K23 were important in the expression of the enterotoxic activity of STb. Dreyfus et al. (1992) described the role of arginine R29 in toxicity. Therefore, these basic residues were tested for their affinity toward SFT using alanine replacement mutagenesis. The binding assay was done in a buffer containing $150 \mathrm{mM} \mathrm{NaCl}$ to more accurately mimic the physiological state. The dissociating salt should also help to neutralize the surface charges found on $\mathrm{STb}$ toxin. Because a significant decrease was observed in binding activity with mutants K22A, K23A and R29A, the individual amino acid residues K22, K23 and R29 evidently have an important and equivalent implication in the attachment to SFT. Yet, K22 and K23 seem to have an important role in binding as the double mutant K22A-K23A exhibited significantly less binding 
capacity to SFT than the double mutant K23A-R29A. Moreover, an additive effect in binding reduction was noted when K22 and K23 were co-mutated. The results obtained for the various mutants tested indicate that binding to SFT represents the initial event leading to the in vivo enterotoxicity observed. In fact, the reduction in toxicity was always either equal to or greater than the decrease in binding to SFT. This was expected, as the binding of a toxin to its receptor is the trigger step in pathogenesis leading to specific toxic consequences. Significantly, the lysine residue K23 seems to have an additional role in enterotoxicity as inferred by the dissociation observed for mutants K23A, K22A-K23A and K23A-R29A between the enterotoxic and binding activities. Discrepancies were noted between our results and those of Fujii et al. (1994), notably about the relevance of K22 for enterotoxicity. Their K22L mutant was shown to be less enterotoxic $(10 \%)$ than the K22A mutant $(70 \%)$ presented in this study. This divergence may be explained by the shorter side chain of the alanine of our K22A mutant compared to the longer leucine of Fujii's K22L mutant.

At position 30 inside the loop (C21-C36), substitution of the aspartate for either alanine or valine reduced the enterotoxicity while the attachment to SFT seemed to be favoured. The suppression of the negative charge present on the aspartate side chain may eliminate a repulsive force that could occur in the vicinity of the SFT-binding site. Indeed, SFT bears an anionic sulfate group. Moreover, the substantial difference observed in attachment between mutants D30A and D30V could result from the preferential presentation of SFT due to the larger size of valine versus alanine or to the more hydrophobic property of valine compared to alanine. In contrast, this mutation appears to alter a subsequent step in enterotoxicity. The mutant D30V reported by Dreyfus et al. (1992) showed a similar enterotoxicity level $(39 \%)$ to the D30A mutant examined in this study $(49 \%)$.

Because of the flexibility conferred by four glycines in the loop (C21-C36), we hypothesized that they would play a role in binding. Toxins such as $\alpha$-haemolysin of $S$. aureus are known to display this characteristic, which facilitates membrane insertion. To test this hypothesis, glycines G24, G27, G31 and G34 were selected for mutagenesis. The results showed a decrease in both attachment and enterotoxicity (except for mutant G34A, which showed a binding of $118 \%$ ). This reduction may be due either to the elimination of the flexibility or to the low space occupancy of the glycine residue. As observed for K23, G31 and G34 seem to play an additional role in enterotoxicity other than simply the attachment. This conclusion is inferred by the dissociation between the enterotoxic and the binding activities observed for mutant G31A ( $1 \%$ and $41 \%$, respectively) and mutant G34A (13\% and $118 \%$, respectively).

Among other SFT-binding proteins, the FasG subunit of the 987P fimbriae was shown to bind to its SFT receptor (Choi \& Schifferli, 1999). Specifically, residues R116,
K117 and K118 in alanine-scanning site-specific mutagenesis experiments have been shown to play a role in attachment to SFT. Thus, for STb and FasG, closely gathered basic residues appeared to interact with SFT, involving a salt-bridge interaction with the sulfate group of SFT (Choi \& Schifferli, 1999). Similar observations were made with properdin, another SFT-binding protein (Holt et al., 1990). In contrast, the flavonol 3-sulfotransferase (ST) displays some similarities with the STb toxin. ST is an enzyme using $3^{\prime}$-phosphoadenosine $5^{\prime}$ phosphosulfate (PAPS) as cosubstrate. Structurally, PAPS is closely related to SFT, both molecules having an acidic sulfate group linked to a sugar ring. Marsolais \& Varin (1995) did site-specific mutagenesis on ST throughout an amino acid stretch known to bind the cosubstrate PAPS, ${ }^{276}$ RKGXXGDWK(N/T)XFT ${ }^{288}$. They demonstrated that residues R276, K277, G281 and K284 were involved in cosubstrate binding by using mutants. Those residues could correspond to K22, K23, G27 and R29, found within STb loop ${ }^{22}$ KKGFLGVRDGTAGA ${ }^{35}$. Marsolais \& Varin (1995) also established that conservative replacements such as K284R have little effect on PAPS-binding activity.

To test the hypothesis concerning the implication of the hydrophobic $\alpha$-helix in enterotoxicity according to the STb NMR structure, three major hydrophobic residues facing the solvent (F37, I41 and M42) were mutated (Sukumar et al., 1995). These mutants demonstrated weak enterotoxicity in the rat loop assay, providing compelling evidence for the role of selected non-polar residues in pathogenesis. Chao \& Dreyfus (1998) have shown that STb toxin promotes leakage of lipid vesicles, indicating local disruption or membrane insertion. Considering that amphipathic and hydrophobic helical structures are required for membrane insertion of the toxin, we postulated that F37, I41 and M42, belonging to the C-terminal hydrophobic $\alpha$-helix, could be involved in the internalization pathway inferred by Chao \& Dreyfus (1997).

Mutants F37K, I41S and M42S showed the same reduction in binding to SFT compared to the reduction in enterotoxicity. However, these results were difficult to reconcile with other results obtained in our laboratory. In fact, Rousset et al. (1998) have shown that the epitope recognized by STb is specifically a galactose residue linked to a sulfated group at position 3. This structure displays no hydrophobic region enabling an interaction with STb. In fact, galactose is comprised of alcohol groups and one hemiacetal group conferring a polar environment. From these observations, theoretically no direct hydrophobic interaction could occur between STb and SFT. To explain the involvement of hydrophobic amino acids in binding to SFT, a model was developed implying an oligomerization process between STb molecules. As demonstrated recently, STb enterotoxin forms hexamers and heptamers through hydrophobic bonds between C-terminal hydrophobic $\alpha$ helix residues F37, I41 and M42 (Labrie et al., 2001). Full $\mathrm{STb}$ structure integrity is necessary for the oligomerization process to take place. We suggest that there 
is basic binding activity between STb and SFT with a $\mathrm{STb}-\mathrm{STb}$ inter-binding process, in part via the hydrophobic $\alpha$-helix. In this case, the propensity of hydrophobic groups to associate due to their repulsion from the aqueous solvent compels STb molecules to interact. This could explain the decrease in attachment observed with polar mutants located in the hydrophobic $\alpha$-helix. Oligomerization is observed for the $\alpha$-toxin of $S$. aureus following a preliminary binding either with a highaffinity protein receptor, or, more commonly, with ubiquitous low-affinity acceptors (phosphatidylcholine and cholesterol). As for STb, the S. aureus $\alpha$-toxin contains a glycine-rich flexible motif (Menestrina \& Ferreras, 1997).

The most prominent result of this study is the establishment of F37, I41 and M42 as important residues for binding and enterotoxicity activities. In addition, we have demonstrated the importance of charged amino acid residues present in the loop (K22, K23, R29 and D30) in the attachment to SFT and confirmed their role in enterotoxicity. Mutations of these residues strongly inhibited both binding and toxic activities. Moreover, these latter hydrophobic residues appear to be more important in both attachment to SFT and enterotoxicity than the previously identified charged amino acids situated in the loop. Taken together, the results indicate that hydrophobic amino acids are directly implicated in the oligomerization process that precedes the binding to SFT. Binding to SFT is due to interaction of SFT with the positively charged lysine and arginine residues of $\mathrm{STb}$. Our study has also clearly established that the binding to SFT is a prerequisite step preceding fluid secretion, as a reduction in binding was concomitantly associated with an equivalent or greater decrease in toxicity.

\section{ACKNOWLEDGEMENTS}

This work was supported by grant OGP0139070 to J.D.D. from the Natural Sciences and Engineering Research Council of Canada (NSERC) and grant 93-ER-0214 from Fonds pour la Formation au Chercheurs et l'Aide à la Recherche (FCAR). V.L. is the recipient of a studentship from FCAR.

We thank M. Tousignant and C. Bérubé for their valuable collaboration with the rat loop assay; F. Dumas for the protein sequencing service and HPLC resourcefulness; and Dr Kornblatt for her helpful advice with CD measurements.

\section{REFERENCES}

Arriaga, Y. L., Harville, B. A. \& Dreyfus, L. A. (1995). Contribution of individual disulfide bonds to biological action of Escherichia coli heat-stable enterotoxin B. Infect Immun 63, 4715-4720.

Beausoleil, H.-E. \& Dubreuil, J. D. (2001). In vitro binding characteristics and affinity for sulfatide of Escherichia coli STb enterotoxin. Receptors and Channels (in press).

Bossé, M., Handl, C. E., Lortie, L. A., Harel, J. \& Dubreuil, J. D. (1993). Fusion of the genes encoding Escherichia coli heat-stable enterotoxin $\mathrm{b}(\mathrm{STb})$ and the maltose-binding protein to obtain mature STb enterotoxin. J Gen Microbiol 139, 631-638.

Chao, K. L. \& Dreyfus, L. A. (1997). Interaction of Escherichia coli heat-stable enterotoxin B with cultured human intestinal epithelial cells. Infect Immun 65, 3209-3217.

Chao, K. L. \& Dreyfus, L. A. (1998). Escherichia coli heat-stable enterotoxin B promotes leakage and fusion of lipid vesicles. 98th General Meeting of ASM, abstract B-268.

Choi, B.-K. \& Schifferli, D. M. (1999). Lysine residue 117 of the FasG adhesin of enterotoxigenic Escherichia coli is essential for binding of 987P fimbriae to sulfatide. Infect Immun 67, 5755-5761.

Cunningham, B. C. \& Wells, J. A. (1989). High-resolution epitope mapping of hGH-receptor interaction by alanine-scanning mutagenesis. Science 244, 1081-1085.

Dreyfus, L. A., Urban, R. G., Whipp, S. C., Slaughter, C., Tachias, K. \& Kupersztoch, Y. M. (1992). Purification of the $\mathrm{ST}_{\mathrm{B}}$ enterotoxin of Escherichia coli and the role of selected amino acids on its secretion, stability and toxicity. Mol Microbiol 6, 2397-2406.

Dreyfus, L. A., Harville, B., Howard, D. E., Shaban, R., Beatty, D. M. \& Morris, S. J. (1993). Calcium influx mediated by the Escherichia coli heat-stable enterotoxin B $\left(\mathrm{ST}_{\mathrm{B}}\right)$. Proc Natl Acad Sci U S A 90, 3202-3206.

Dubreuil, J. D. (1997). Escherichia coli STb enterotoxin. Microbiology 143, 1783-1795.

Dubreuil, J. D., Fairbrother, J. M., Lallier, R. \& Larivière, S. (1991). Production and purification of heat-stable enterotoxin $b$ from a porcine Escherichia coli strain. Infect Immun 59, 198-203.

Fujii, Y., Okamuro, Y., Hitotsubashi, S., Saito, A., Akashi, N. \& Okamoto, K. (1994). Effect of alterations of basic amino acid residues of Escherichia coli heat-stable enterotoxin II on enterotoxicity. Infect Immun 62, 2295-2301.

Fujii, Y., Kondo, Y. \& Okamoto, K. (1995). Involvement of prostaglandin E2 synthesis in the intestinal secretory action of Escherichia coli heat-stable enterotoxin II. FEMS Microbiol Lett 130, 259-265.

Fujii, Y., Nomura, T., Yamanaka, H. \& Okamoto, K. (1997). Involvement of $\mathrm{Ca}^{2+}$-calmodulin-dependent protein kinase II in the intestinal secretory action of Escherichia coli heat-stable enterotoxin II. Microbiol Immunol 41, 633-636.

Goldstein, J. L., Sahi, J., Bhuva, M., Layden, T. J. \& Rao, M. C. (1994). Escherichia coli heat-stable enterotoxin-mediated colonic $\mathrm{Cl}^{-}$secretion is absent in cystic fibrosis. Gastroenterology 107, 950-956.

Handl, C. E., Harel, J., Flock, J. I. \& Dubreuil, J. D. (1993). High yield of active STb enterotoxin from a fusion protein (MBP-STb) expressed in Escherichia coli. Protein Expr Purif 4, 275-281.

Hitotsubashi, S., Fujii, Y., Yamanaka, H. \& Okamoto, K. (1992). Some properties of purified Escherichia coli heat-stable enterotoxin II. Infect Immun 60, 4468-4474.

Holt, G. D., Pangburn, M. K. \& Ginsburg, V. (1990). Properdin binds to sulfatide and has a sequence homology with other proteins that binds to sulfate glycoconjugates. $J$ Biol Chem 265, 2852-2855.

Kaper, J. B., Morris, J. G., Jr \& Levine, M. M. (1995). Cholera. Clin Microbiol Rev 8, 48-86.

Kennedy, D. J., Greenberg, R. N., Dunn, J. A., Abernathy, R., Ryerse, J. S. \& Guerrant, R. L. (1984). Effects of Escherichia coli heat-stable enterotoxin $\mathrm{STb}$ on intestines of mice, rats, rabbits, and piglets. Infect Immun 46, 639-643.

Kupersztoch, Y. M., Tachias, K., Moomaw, C. R., Dreyfus, L. A., Urban, R., Slaughter, C. \& Whipp, S. (1990). Secretion of methanolinsoluble heat-stable enterotoxin (STB): energy- and SecAdependent conversion of pre-STB to an intermediate indistinguishable from the extracellular toxin. J Bacteriol 172, 2427-2432. 
Labrie, V., Harel, J. \& Dubreuil, J. D. (2001). Oligomerization of Escherichia coli enterotoxin b through its C-terminal hydrophobic alpha-helix. Biochim Biophys Acta 1535, 128-133.

Lala, A. K. \& Raja, S. M. (1995). Photolabeling of a pore-forming toxin with the hydrophobic probe $2-\left[{ }^{3} \mathrm{H}\right]$ diazofluorene. $J$ Biol Chem 270, 11348-11357.

Marsolais, F. \& Varin, L. (1995). Identification of amino acid residues critical for catalysis and cosubstrate binding in the flavonol 3-sulfotransferase. J Biol Chem 270, 30458-30463.

Menestrina, G. \& Ferreras, M. (1997). Alpha-toxin (Staphylococcus aureus). In Guidebook to Protein Toxins and Their Use in Cell Biology, pp. 10-13. Edited by R. Rappuoli \& C. Montecucco. New York: Oxford University Press.

Okamoto, K., Baba, T., Yamanaka, H., Akashi, N. \& Fujii, Y. (1995). Disulfide bond formation and secretion of Escherichia coli heatstable enterotoxin II. J Bacteriol 177, 4579-4586.

Peterson, J. W. \& Whipp, S. C. (1995). Comparison of the mechanisms of action of cholera toxin and the heat-stable enterotoxins of Escherichia coli. Infect Immun 63, 1452-1461.

Rousset, É., Harel, J. \& Dubreuil, J. D. (1998). Sulfatide from the pig jejunum brush border epithelial cell surface is involved in binding of Escherichia coli enterotoxin b. Infect Immun 66, 5650-5658.

Spangler, B. D. (1992). Structure and function of cholera toxin and the related Escherichia coli heat-labile enterotoxin. Microbiol Rev 56, 622-647.

Sukumar, M., Rizo, J., Wall, M., Dreyfus, L. A., Kupersztoch, Y. M. \& Gierasch, L. M. (1995). The structure of Escherichia coli heatstable enterotoxin b by nuclear magnetic resonance and circular dichroism. Protein Sci 4, 1718-1729.

Ward, R. J., Palmer, M., Leonard, K. \& Bhakdi, S. (1994). Identification of a putative membrane-inserted segment in the alpha-toxin of Staphylococcus aureus. Biochemistry 33, 7477-7484.

Weikel, C. S. \& Guerrant, R. L. (1985). STb enterotoxin of Escherichia coli: cyclic nucleotide-independent secretion. In Microbial Toxins and Diarrhoeal Disease, pp. 94-115. Edited by R. Evered \& J. Whelan. London: Pittman, Ciba Foundation Symposium.

Received 1 March 2001; revised 21 June 2001; accepted 10 July 2001. 